\title{
Shift in prevalence of HPV types in cervical cytology specimens in the era of HPV vaccination
}

\author{
SONJA FISCHER ${ }^{1}$, MARCUS BETTSTETTER ${ }^{2}$, ANDREA BECHER ${ }^{1}$, \\ MARLENE LESSEL ${ }^{1,2}$, CYRIL BANK ${ }^{1}$, MATTHIAS KRAMS ${ }^{3}$, INGRID BECKER ${ }^{3}$, \\ ARNDT HARTMANN $^{4}$, WOLFGANG JAGLA ${ }^{1}$ and ANDREAS GAUMANN ${ }^{1,2}$ \\ ${ }^{1}$ Institute of Pathology Kaufbeuren-Ravensburg, D-87600 Kaufbeuren; \\ ${ }^{2}$ Part Shared Practice Molecular Pathology South Bavaria, D-81675 Munich; ${ }^{3}$ Institute of Pathology Rosenheim, \\ D-83022 Rosenheim; ${ }^{4}$ Institute of Pathology Erlangen, D-91054 Erlangen, Germany
}

Received February 24, 2015; Accepted December 22, 2015

DOI: $10.3892 / 01.2016 .4668$

\begin{abstract}
The aim of the present population-based cohort study was to analyze the association between the prevalence of 32 types of human papilloma virus (HPV) in 615 female patients with abnormal cervical cytopathology findings. In total, 32 HPV types were screened by DNA array technology. HPV infection was detected in 470 women (76.42\%), 419 of whom $(89.15 \%)$ were infected with $\geq 1$ high-risk (HR)-HPV type. HPV16, which is recognized as the main HR-HPV type responsible for the development of cervical cancer, was observed in $32.98 \%$ of $\mathrm{HPV}^{+}$participants, followed by HPV42 (18.09\%), HPV31 (17.66\%), HPV51 (13.83\%), HPV56 (10.00\%), HPV53 (8.72\%) and HPV66 (8.72\%). The prevalence of HR-HPV types, which may be suppressed directly (in the case of HPV16 and 18), or possibly via cross-protection (in the case of HPV31) following vaccination, was considerably lower in participants $\leq 22$ years of age (HPV16, 28.57\%; HPV18, 2.04\%; HPV31, 6.12\%), compared with participants 23-29 years of age (HPV16, 45.71\%; HPV18, $7.86 \%$; HPV31, 22.86\%), who were less likely to be vaccinated. Consequently, the present study hypothesizes that there may be a continuous shift in the prevalence of HPV types as a result of vaccination. Furthermore, the percentage of non-vaccine HR-HPV types was higher than expected, considering that eight HPV types formerly classified as 'low-risk' or 'probably high-risk' are in fact HR-HPV types. Therefore, it may be important to monitor non-vaccine HPV types in future studies, and an investigation concerning several HR-HPV types as risk factors for the development of cervical cancer is required.
\end{abstract}

Correspondence to: Dr Andreas Gaumann, Institute of Pathology Kaufbeuren-Ravensburg, 6 Dr Gutermann Street, D-87600 Kaufbeuren, Germany

E-mail: andreas.gaumann@pathologie-kaufbeuren.de

Key words: human papilloma virus, cervical cancer, high-risk HPV, HPV16, squamous intraepithelial lesions

\section{Introduction}

Human papilloma viruses (HPVs) are small non-enveloped DNA viruses that belong to the papilloma virus family, and infect cutaneous and mucosal epithelia in humans (1). HPVs are extremely common worldwide, and consist of $>170$ types (2). A persistent infection with certain mucosa-tropic types of HPV is recognized as the major factor in the etiology of cervical cancer (3-6). According to the World Health Organization (WHO), cervical cancer is the second most common type of cancer in women, with $~ 530,000$ newly diagnosed cases every year (7). The incidence of cervical cancer in Germany is one of the highest among Western countries, and it is currently the second cause of mortality among women $>50$ years of age (8).

HPV is usually acquired via sexual transmission, and may induce the development of cervical cancer within several years following a persistent infection (9). The progress of cervical cancer is slow, commencing from cervical intraepithelial neoplasia (CIN) (3) and ending with invasive cancer. Precancerous lesions may be effectively detected by cervical screening using the Papanicolaou test (Pap smear). HPVs have been classified into high-risk types (HR-HPVs), which are oncogenic or have oncogenic potential, and low-risk types (LR-HPVs), which do not exhibit a causal association with cancer (9). A recent study provided biological evidence of carcinogenicity for HPV types 26, 53, 66, 67, 68, 70, 73 and 82 , which were previously classified as possibly carcinogenic (HPV26, 53, 66, 68, 73 and 82) and non-carcinogenic (HPV67 and 70) (10). Whereas LR-HPV types account for non-fatal conditions, including warts and condylomas, numerous studies have demonstrated the causal association between HR-HPV and malignant lesions $(4,6,11)$. In a retrospective cross-sectional worldwide study concerning the association between HPV genotype and cervical cancer using data from $>10,000$ patients, HPV DNA was identified in $85 \%$ of patients with cervical cancer (12). The highest carcinogenic risk is attributed to the following HR-HPV types: 16, 18, 31, $33,35,52$ and 58 (13). HPV16 and 18 are associated with $>2 / 3$ of all cervical cancer cases worldwide (14-16), which has led to the development of prophylactic vaccines against these two HPV types (17). 
Currently, there are two approved HPV vaccines in Germany (18). These are the quadrivalent vaccine Gardasil ${ }^{\circledR}$ (Merck \& Co., Inc., Whitehouse Station, NJ, USA), which targets HPV6, 11, 16 and 18, and the bivalent vaccine Cervarix $^{\circledR}$ (GlaxoSmithKline, Brentford, UK), which targets HPV16 and 18. Since 2006, numerous countries have implemented vaccination programs using these two vaccines (18). In Germany, HPV vaccination is usually performed during a routine adolescent health check-up termed $\mathrm{J} 1$, which is available for 12-14 year-old girls, and the recommendation of the Standing Committee on Vaccination at the Robert Koch Institute is to vaccinate between the ages of 9 and 14 years (19). The results from clinical trials indicate that vaccinated girls and women have an increased protection against the development of CIN (20-23). In addition to the proven efficacy against HPV16 and $18(10,15)$, the above bivalent and quadrivalent HPV vaccines are known to confer cross-protection against certain non-vaccine HPV types. The quadrivalent vaccine has been described to cross-protect mainly against HPV31 (24), while the bivalent vaccine has been demonstrated to exhibit a protective effect against HPV31, 33, 45 (25) and 51 (26).

With the increasing impact of vaccination against certain HPV types in young women (27), it is important to monitor shifts in the prevalence of HPV types. Therefore, the aim of the present study is to determine and evaluate the prevalence of different HPV types in Southern Bavaria, and to analyze the local HPV type distribution in women with abnormal cytological diagnostic findings. Cervical co-infection with multiple HPV types is widespread (28), and may contribute to a higher risk of developing cervical lesions with precancerous potential. Consequently, evaluating the prevalence of infections with $>1 \mathrm{HPV}$ type is an additional aim of the present study.

\section{Materials and methods}

Patient cohort. The study cohort consisted of 615 Caucasian women aged between 16 and 93 years. The participants had undergone routine cytological evaluation in three Southern Bavaria pathology centers (Institutes of Pathology at Kaufbeuren, Ravensburg and Rosenheim, Germany) using the Second Munich Cytological Classification (29). The cytology was re-evaluated in the present study using the Bethesda classification system (30). Only data from participants with a conspicuous cervical cytological indication and available HPV analysis were included in the present study. Complete data on the individual vaccination status of every participant was not available. Written informed consent was obtained from all patients for the use of their data. The present study was approved under the ethical regulations that exist at the Medical Faculty of the University of Regensburg (Bavaria, Germany).

Cytology. Cytological specimens were collected between December 2010 and September 2014 by Pap smear. The specimens were dehydrated, and subsequently stained with Harris' hematoxylin (Carl Roth GmbH, Karlsruhe, Germany), bleached with hydrated hydrochloric acid (Hernicht GmbH, Sulzberg, Germany), and stained orange-red with Papanicolaou's staining solution Orange G (EA 50; VWR International $\mathrm{GmbH}$, Darmstadt, Germany), according to the manufacturer's protocol. The slides were reviewed by qualified pathologists at the Institutes of Pathology at Kaufbeuren, Ravensburg and Rosenheim using the Bethesda classification system (31). Smears with atypical squamous cells of undetermined significance (ASC-US), low-grade squamous intraepithelial lesion (LSIL) and high-grade squamous intraepithelial lesion (HSIL) were subjected to further analysis.

Histopathology. In addition to the Pap smear, cervical conization specimens were obtained from a subgroup of 86 out of 615 participants. The cervical conization specimens were reviewed and classified as grades CIN1, CIN2 and CIN3, according to the WHO criteria (32). The specimens were immediately fixed following surgery in buffered $4 \%$ formaldehyde (Hernicht $\mathrm{GmbH}$ ), and subsequently analyzed with hematoxylin and eosin staining (VWR International $\mathrm{GmbH})$. Tissue sections (2- $\mu \mathrm{m}$ thick; RM2235 Microtome; Leica Microsystems GmbH, Wetzlar, Germany) were obtained from paraffin-embedded tissue (Hasuwax Paraffin; Sussmann \& Steinhauser GmbH, Kaufbeuren, Germany). In certain tissues, p16 immunohistochemistry was performed using the CINtec ${ }^{\circledR}$ Histology kit (catalog no., 9517; mouse monoclonal anti-p16 antibody; dilution, 1:50; Roche Diagnostics $\mathrm{GmbH}$, Mannheim, Germany), according to the manufacturer's protocol, to confirm cervical intraepithelial dysplasia.

HPV DNA testing. DNA was isolated from the formalin-fixed, paraffin-embedded (FFPE) tissues using the QIAamp DNA FFPE Tissue kit (Qiagen GmbH, Hilden, Germany) and the QIAcube (Qiagen $\mathrm{GmbH}$ ), according to the manufacturer's protocol. HPV testing was performed using a DNA-based liquid-crystal display (LCD)-Array kit (LCD Array HPV 3.5; Chipron GmbH, Berlin, Germany), which contained 32 specific capture probes for the identification of 32 types of HPV. In total, $20 \mu \mathrm{l}$ polymerase chain reaction (PCR) AmpliTaq Gold ${ }^{\circledR} 360$ Master Mix (Applied Biosystems; Thermo Fisher Scientific, Inc., Waltham, MA, USA) was used, which contained $1.2 \mu \mathrm{l}$ pre-labeled primer mix, $0.2 \mu \mathrm{M}$ dNTPs, $2 \mathrm{mM}$ magnesium, $0.2 \mathrm{U}$ Taq-Gold polymerase and $2 \mu \mathrm{l}$ template DNA. Amplification was performed in a DNA Engine ${ }^{\circledR}$ Thermal Cycler (Bio-Rad Laboratories, Inc., Hercules, CA, USA) with the following PCR program: Initial denaturation of $10 \mathrm{~min}$ at $95^{\circ} \mathrm{C}$ followed by 42 cycles of $1 \mathrm{~min}$ at $94^{\circ} \mathrm{C}, 1.5 \mathrm{~min}$ at $45^{\circ} \mathrm{C}$ and $1.5 \mathrm{~min}$ at $72^{\circ} \mathrm{C}$, with a final elongation of $3 \mathrm{~min}$ at $72^{\circ} \mathrm{C}$. The present study adapted the classification of HR- and LR-HPV types according to previous studies concerning the carcinogenic properties of particular HPV types $(10,33)$. Therefore, the panel of identifiable HPV types consisted of $20 \mathrm{HR}-\mathrm{HPV}$ types (HPV16, 18, 31, 33, 35, 39, 45, 51, 52, 53, 56, 58, 59, 66, $67,68,70,73,82$ and 83) and 12 LR-HPV types (HPV6, 11, 42, $44,54,61,62,72,81,84,87,90$ and 91). The positive control contained DNA from a HPV-positive (HPV 16) sample while the negative control contained no DNA. Using the LCD-Array kit (LCD Array HPV 3.5; Chipron GmbH), the labeled PCR fragments were combined with hybridization buffer (Chipron $\mathrm{GmbH}$ ) and hybridized at $35^{\circ} \mathrm{C}$ for $30 \mathrm{~min}$ to the individual array fields of one chip with specific capture probes (Chipron $\mathrm{GmbH}$ ). Following a washing procedure according to the manufacturer's protocol, each field was incubated with a 


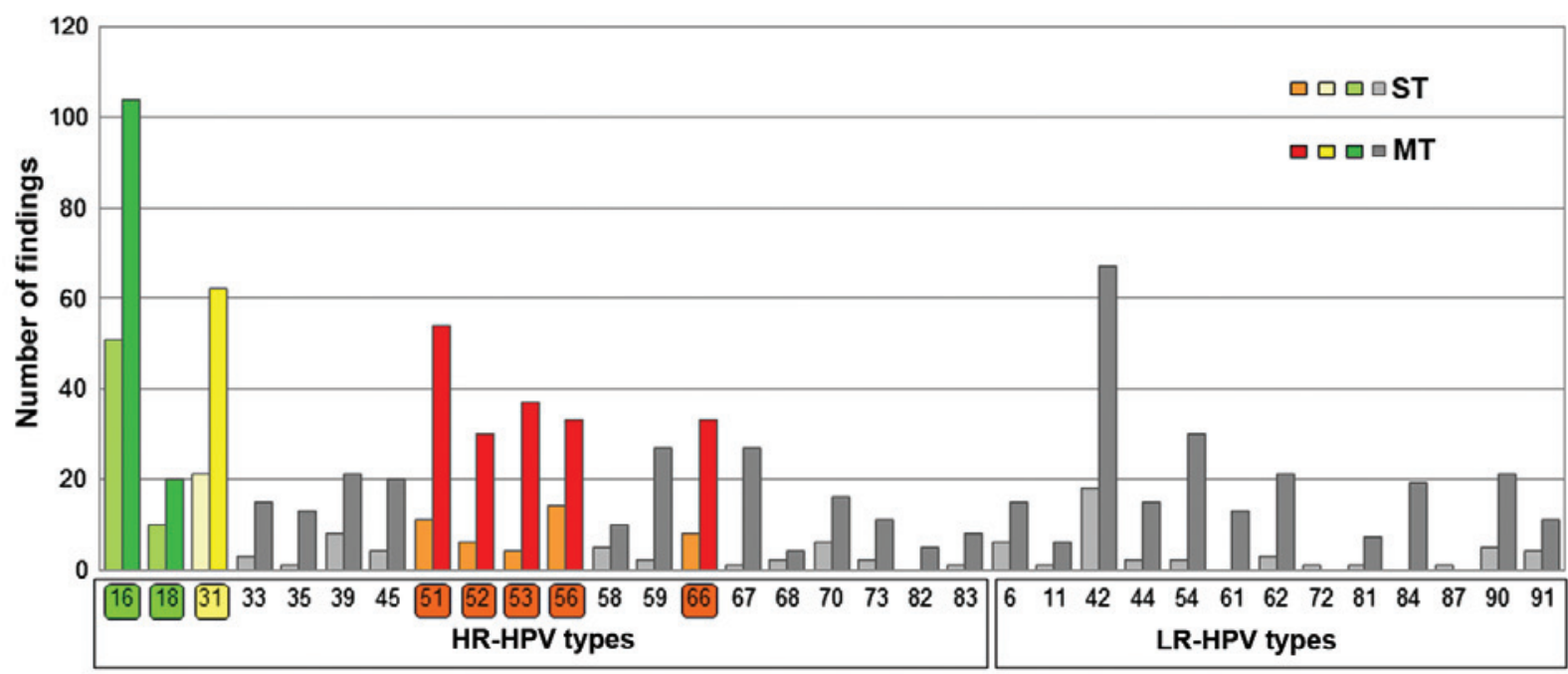

Figure 1. Prevalence of HR- and LR-HPV types among participants in the present study, represented according to their incidences as ST or MT infection. HPV types targeted directly by vaccination are labelled green. HPV types where a cross-protective effect has been described for current vaccines are labelled yellow. HR-HPV types without vaccination coverage and a high incidence are labelled red. HPV types were identified using Chipron LCD Array HPV 3.5 or DNA sequencing (in the case of HPV87). HPV, human papilloma virus; HR, high risk; LR, low risk; ST, single type; MT, multiple type; LCD, liquid-crystal display.

secondary label solution for $5 \mathrm{~min}$ at room temperature (enzyme-conjugate, Chipron $\mathrm{GmbH}$ ). Subsequent to a second washing step, the bound PCR fragments were visualized by a blue precipitate formed by the 'BLUE stain' enzyme substrate provided (Chipron $\mathrm{GmbH}$ ).

PCR results were evaluated using SlideReader Software v2.0 (Chipron $\mathrm{GmbH}$ ). PCR, hybridization, labeling and staining were performed according the manufacturer's protocol (Chipron GmbH). HPV types that were detected by PCR but did not generate signals on the LCD array were sequenced and subjected to Basic Local Alignment Search Tool analysis (National Center for Biotechnology Information, Bethesda, MD, USA). One tissue sample with HPV87 was detected by sequencing.

\section{Results}

Patient characteristics. The current study consisted of 615 female participants with abnormal cervical cytological pathology (ASC-US, LSIL or HSIL). In total, 35.61\% (219/615) of the participants were $<30$ years of age, $32.36 \%(199 / 615)$ were $30-44$ years old, $28.62 \%$ (176/615) were $45-59$ years old and $3.41 \%(21 / 615)$ were $>60$ years of age. In $470(76.42 \%)$ of these participants, HPV infection was detected, and $419(89.15 \%)$ of them were infected with $\geq 1$ HR-HPV type. As shown in Table I, a total of 204 out of 615 participants (33.17\%) were infected with a single type (ST) of HPV, while 266 participants $(43.25 \%)$ were infected with multiple types (MTs), providing a total of 775 HPV results (mean of participants with MT infection, 2.9 types). The distribution of the detected HPV types divided by age group ( $<30$ years; $30-44$ years; $45-59$ years; and $\geq 60$ years) is revealed in Table I. The incidence rates in Table I are presented as the total number of participants and the number of $\mathrm{HPV}^{+}$participants. The prevalence of infected participants declined by age $(<30$ years, $86.30 \%$; $30-44$ years, $77.89 \%$; $45-59$ years, $63.31 \%$ ). In participants $\geq 60$ years old, there was an unanticipated high rate $(71.43 \%)$ of infection.
However, this is not significant, due to the small group size $(n=21)$.

The present study additionally analyzed participants with ST or MT infection. The highest rate of MT infection (64.02\% of all infected patients) was observed in participants $<30$ years old. In participants $>30$ years old, the percentage of patients with MT infection was lower (30-44 years, $51.61 \%$; $45-59$ years, $52.25 \%$; $\geq 60$ years, $46.67 \%$ ). The highest prevalence of HPV type was HPV16 (HR), which was present in $32.98 \%$ of participants that tested $\mathrm{HPV}^{+}$, followed by HPV42 (LR; 18.09\%), HPV31 (HR; 17.66\%), HPV51 (HR; 13.83\%), HPV56 (HR; 10.00\%), HPV66 (HR; 8.72\%) and HPV53 (HR; 8.72\%). HR-HPV type infections that may be directly prevented by vaccination or via cross-protection with available vaccines are marked in green (HPV16 and 18) and yellow (HPV31) in Figs. 1 and 2, while HR-HPV types with the highest prevalence (HPV51, 53, 56 and 66), with no protection from vaccinations, are marked in red. HPV18 infection occurred in $6.38 \%$ of participants that tested $\mathrm{HPV}^{+}$. The prevalence of HPV16 was highest in participants $<30$ years $(41.27 \%)$, and declined with older age (30-44 years, $30.97 \%$; 45-59 years, $22.52 \%$; $\geq 60$ years, $26.67 \%$ ).

Incidence of ST and MT infection. Fig. 1 represents the prevalence of ST and MT infections for all the HPV types analyzed. HPV16 and 18, known to be cervical carcinogens, were observed in $39.36 \%$ of $\mathrm{HPV}^{+}$participants (ST or MT infection) and in $44.05 \%$ of HR-HPV infected participants. In $67.10 \%$ (104/155) of participants, the presence of HPV16 was associated with MT infection, while this percentage was $66.67 \%$ (20/30) for HPV18. The most frequent HPV types that exhibited co-infection with HPV16 were HPV42 (n=22), HPV51 ( $n=21)$, HPV31 (n=17), HPV66 (n=16), HPV56 (n=12), HPV67 (n=12), HPV18 $(n=11), \operatorname{HPV} 39(n=11)$ and HPV90 $(n=11)$. In total, $55.95 \%$ of HR-HPV-infected participants were not infected with HPV16 or 18 . The less prevalent HPV18 was identified in 20 out of 30 participants with MT-HPV infection, most 


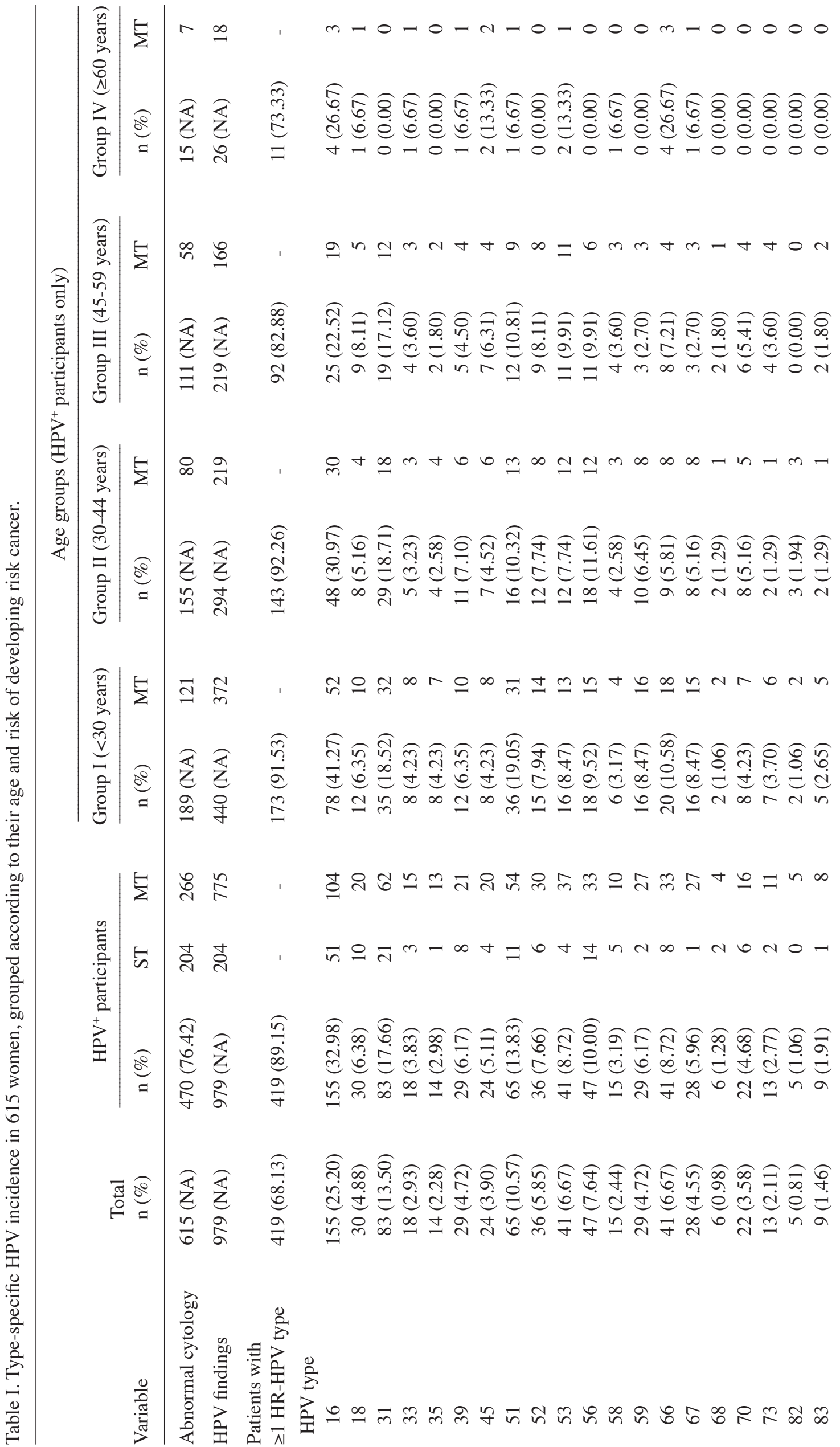




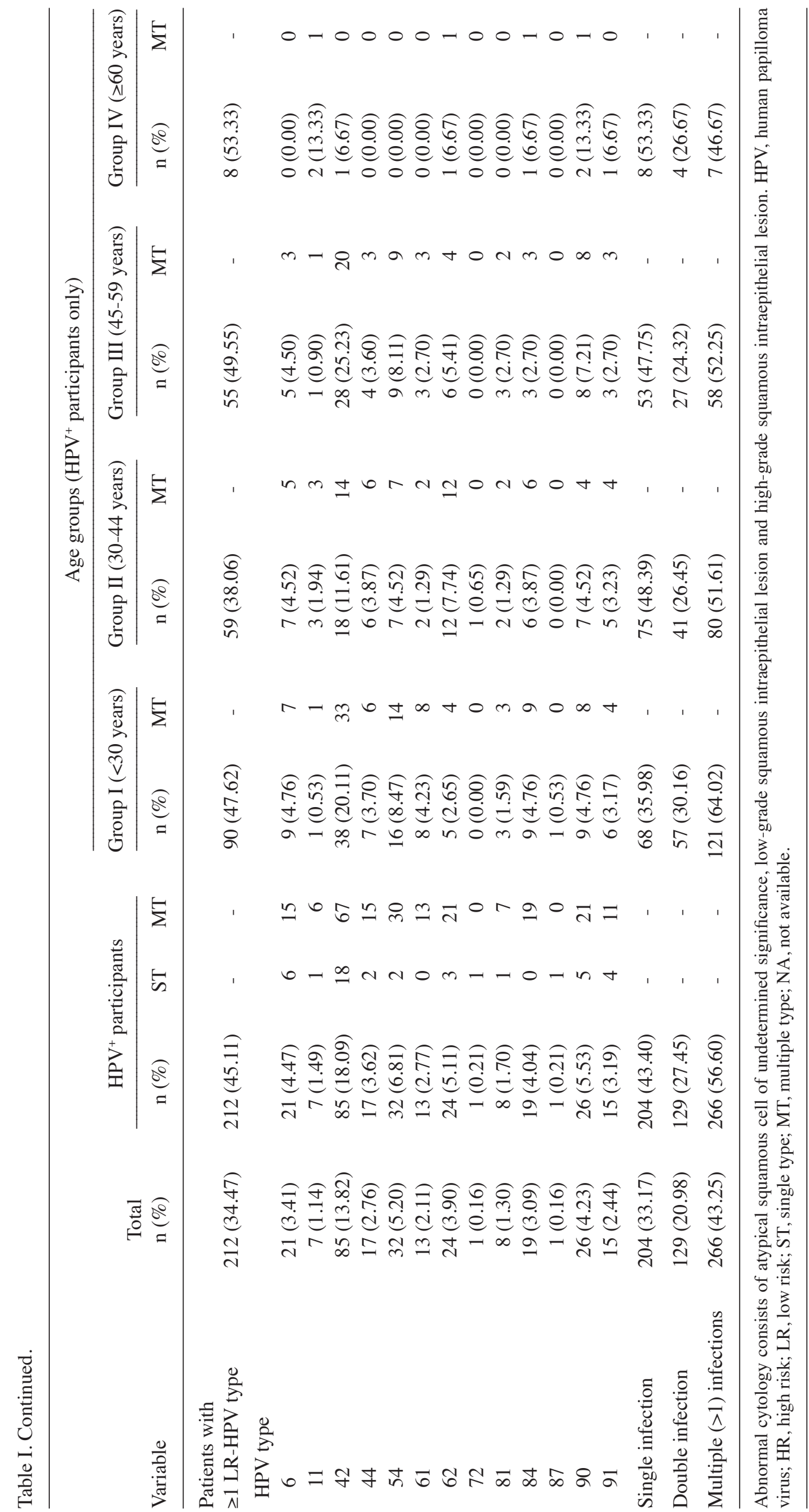




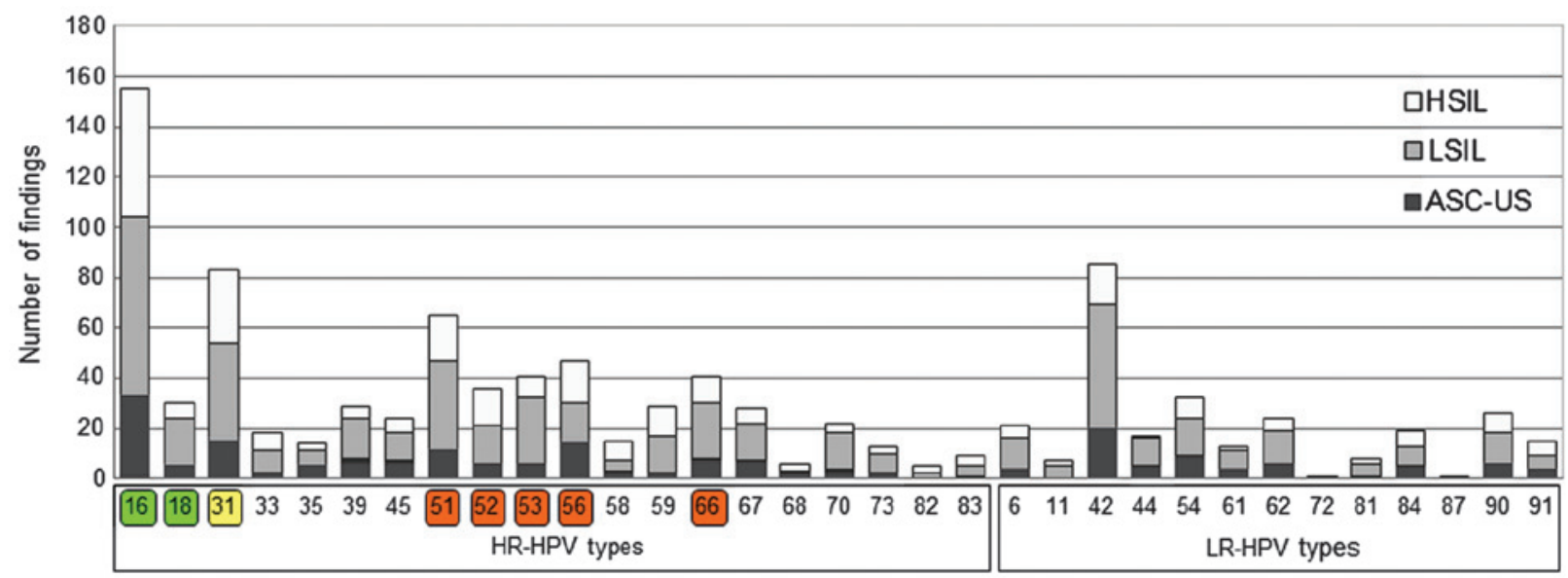

Figure 2. Prevalence of HPV types among participants in the present study with ASC-US, LSIL or HSIL. HPV types targeted directly by vaccination are labelled green. HPV types where a cross-protective effect has been described for current vaccines are labelled yellow. HR-HPV types without vaccination coverage and high incidence are labelled red. HPV types were identified using Chipron LCD Array HPV 3.5 or DNA sequencing (in the case of HPV87). HPV, human papilloma virus; ASC-US, atypical squamous cell of undetermined significance; LSIL, low-grade squamous intraepithelial lesion; HSIL, high-grade squamous intraepithelial lesion; HR, high risk; LR, low risk; LCD, liquid-crystal display.

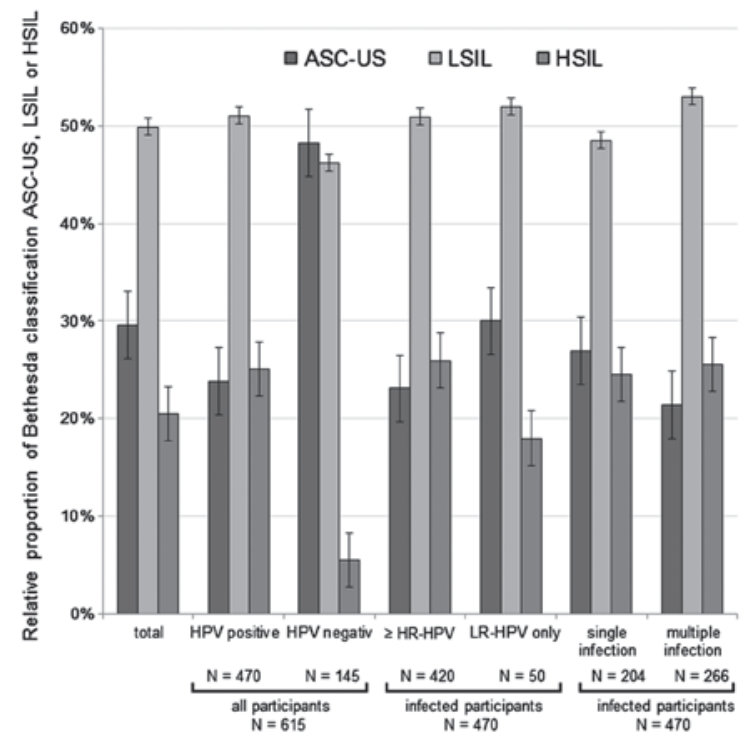

Figure 3. Relative distribution of $\mathrm{HPV}^{+}$and $\mathrm{HPV}^{-}$participants according to the Bethesda cervical cytological classification (ASC-US, LSIL or HSIL). The distribution of cervical cytological classification among participants in the present study with $\geq 1$ HR-HPV or 1 probably HR-HPV type with additional LR-HPV appearance, and the distribution of cervical cytological classification among participants with $\geq 1$ LR-HPV type without any HR-HPV appearance, are depicted. Furthermore, the relative distribution of participants according to single or multiple infection is represented in the graph. HPV, human papilloma virus; ASC-US, atypical squamous cell of undetermined significance; LSIL, low-grade squamous intraepithelial lesion; HSIL, high-grade squamous intraepithelial lesion; HR, high risk; LR, low risk.

frequently with HPV16 ( $\mathrm{n}=11)$, and rarely with HPV51 $(\mathrm{n}=5)$, HPV31 (n=3), HPV44 (n=3), HPV59 (n=3) and HPV66 (n=3).

HPV type and association with cervical cancer. Fig. 2 represents the number of findings of each HPV type, and the corresponding cervical cytological finding, based on the Bethesda classification system (30). The relative distribution of cytological diagnosis in the group of $\mathrm{HPV}^{+}$participants was clearly different, compared with the cytological diagnosis of $\mathrm{HPV}^{-}$participants (Fig. 3). The prevalence of LSIL and HSIL was increased in the group of $\mathrm{HPV}^{+}$participants, which suggests that HPV may cause the development of a precancerous lesion. The distribution of cytological findings among participants with $\geq 1$ HR-HPV type, partially with an additional LR-HPV appearance, differed from the distribution of cytological findings among participants with $\leq 1$ LR-HPV type and without any HR-HPV appearance. Therefore, this suggests that there were proportionally more LSIL and HSIL cases, compared with ASC-US cases. Three HR-HPV types, namely HPV16, 18 and 31 were present singularly in $65.25 \%$ of HSIL cases, compared with 37.79 and $26.37 \%$ in LSIL and ASC-US cases, respectively. The general distribution of cytological diagnosis according to HPV type, HR- or LR-HPV prevalence and ST or MT infection status are presented in Fig. 3 (ASC-US, n=182; LSIL, n=307; HSIL, $\mathrm{n}=126)$. There were proportionally more participants with MT-HPV infection classified as LSIL and HSIL, compared with participants with a single infection, who were classified as ASC-US. Notably, 7 out of 266 participants (2.63\%) with MT possessed LR-HPV types and, in contrast, 259 women (97.37\%) possessed HR-HPV types or a mixture of HR- and LR-HPV types.

Cervical conization specimens. In a subgroup of 86 participants, cervical conization specimens were obtained, and 80 of these participants exhibited cervical intraepithelial neoplasia, which were classified as CIN1 (22/80), CIN2 (27/80) or CIN3 (31/80). The prevalence of HR-HPVs was associated with a more severe cervical intraepithelial neoplasia (HR-HPV appearances: CIN1, 77.27\%; CIN2, 81.48\%; and CIN3, 90.32\%). The total prevalence of HR-HPV types 16 , 18 and 31 was highest in participants whose neoplasms were classified as CIN3 (64.52\%; 20/31), followed by $55.56 \%(15 / 27)$ of neoplasms classified as CIN2 and $50.00 \%$ $(11 / 22)$ of neoplasms classified as CIN1. By contrast, there was no evidence that MT infections were more prevalent in neoplasms classified as CIN3 (48.39\%), compared with those classified as CIN2 (50.85\%). In addition, the present 

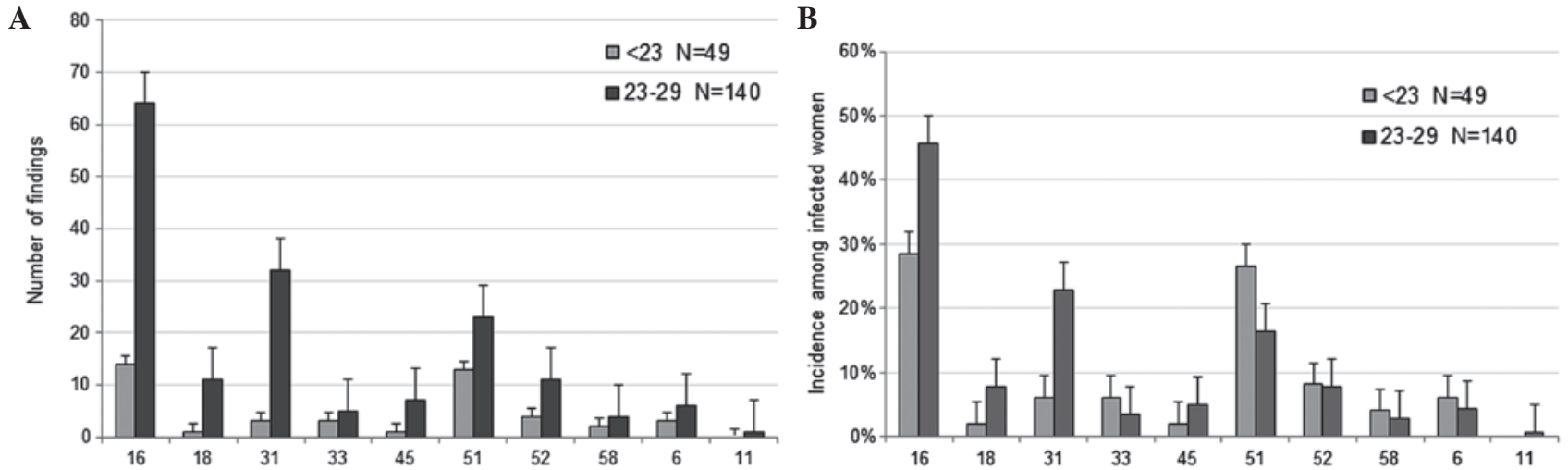

Figure 4. (A) Number of findings and (B) incidences of HPV types that are impaired directly by vaccination or potentially via cross-protection by available vaccines. Participants $<30$ years of age are divided into two subgroups (group $1,<23$ years old; group 2, 23-29 years old), where women $\leq 22$ years old are more often protected against certain HPV types by vaccination, compared with participants aged $>23$ years old. HPV, human papilloma virus.

study demonstrated that in participants with CIN3 lesions, a cytological diagnosis of ASC-US was present in $19.35 \%$ of participants, LSIL in $32.26 \%$ participants and HSIL in $48.39 \%$ of participants. Participants that possessed CIN1 or CIN2 neoplasms were more likely to exhibit LSIL $(50.00 \%$; $66.67 \%)$, compared with HSIL $(31.82 \% ; 18.52 \%)$. There was no accumulation of specific HPV types among the non-vaccinated HR-HPV participants that could be assigned to one of the CIN classes.

HPV vaccination. In Germany, a routine $\mathrm{HPV}$ vaccine has been recommended for 12-17 year-old girls since 2007 (34). Consequently, in the present study, participants aged 19-21 years may have been vaccinated, while women $>22$ years presumably had not been vaccinated. Therefore, a disparity was expected among the participants in the $<30$-year-old group. As a result, this age group was divided into two subgroups: Those aged $<23$ years $(n=49)$, and those aged 23-29 years $(n=140)$. For participants $<23$ years old, information on vaccination status was obtained from 24 participants, and the information was as follows: $37.50 \%$ of patients $(9 / 24)$ were vaccinated $\left(\right.$ Gardasil $^{\circledR}, 7$; unknown, $2)$, while $62.50 \%(15 / 24)$ were not vaccinated. Although the present study did not include data on the individual vaccination status of all participants, based on the results from the 9 participants in the $<23$-year-old group, it was estimated that $\sim 1 / 3$ of women $<23$ years of age were protected against HPV by vaccination. The number of HPV types that may be suppressed directly by vaccination (HPV6, 11, 16 and 18) or potentially reduced via cross-protection by $\geq 1$ of the current vaccines (HPV31, 33, 45, 52 and 58) are presented in Fig. 4. The prevalence of HPV16, 18 and 31 was considerably decreased in participants aged $<23$ years old (HPV16, 28.57\%; HPV 18, 2.04\%; HPV31, 6.12\%), compared with those aged 23-29 years old (HPV16, 45.71\%; HPV18, 7.86\%; HPV31, 22.86\%). These results support that vaccination of young women is important in preventing infections with HR-HPV. However, the prevalence of certain HR-HPVs that are not affected by direct vaccination or cross-protection remains quite high. The most frequent HR-HPVs identified by the present study were HPV51 (13.83\%), HPV56 (10.00\%) and HPV66 (8.72\%), which are labeled red in Figs. 1 and 2.

\section{Discussion}

The present study evaluated the prevalence of important HPV types in a local cohort of participants from Southern Bavaria, and analyzed the HPV type distribution in participants with abnormal cytological diagnostic findings, who were separated by various age groups. It is well known that the distribution of HPV types varies around the world (14), and it is hypothesized, based on a pooled analysis from women with normal cytological findings in various studies, that the prevalence of HPV16 is more prominent in Europe and North America, compared with other regions of the world $(12,14,33,34)$. In the current study, HPV infection was detected in $76.42 \%$ of participants with an abnormal cytological finding. In total, $89.15 \%$ of participants were infected with $\geq 1 \mathrm{HR}-\mathrm{HPV}$, which is significantly higher than the incidence previously reported in a similar study conducted in Italy (68.90\%) (35). However, it taken into consideration the fact that the present study classified more HPV types as HR-HPV than the aforementioned previous study did. In the present study, an outdated HPV classification system with several probably HR-HPV types identified that the HR-HPV prevalence was $81.50 \%$ in participants $<30$ years of age. This is slightly lower than the prevalence previously described for other regions in Germany (88.40\%) (36). However, using a classification system based on novel biological evidence, which revealed that more HPV types exhibit carcinogenicity than previously acknowledged $(10,33)$, the prevalence of HR-HPV types identified by the present study increased to $91.53 \%$.

In addition, the present study observed that the prevalence of HPV16 and 18 in participants $>30$ years old was $33.81 \%$, which is close to the prevalence of $34.50 \%$ identified in a previous study (36). In participants aged $<30$ years old, the highest incidence of HPV16 and 18 was $47.62 \%$, which is higher than the prevalence of $37.40 \%$ identified in a previous study on young women $<30$ years of age in Germany (37). The decreasing prevalence of HPV16 in older participants demonstrated by the current study has previously been described in a cross-national study conducted prior to the initiation of the HPV vaccination program (11).

It was previously demonstrated that MT HPV are more common in women with cervical lesions, compared with 
women with normal cervical cytology (38). However, it has been observed that the presence of MT HPV does not affect the severity of infection, and HPV infections are independent of one another (39). In the present study, $43.40 \%$ of all $\mathrm{HPV}^{+}$ participants were infected with one type of HPV (ST HPV), whereas the majority of participants exhibited MT HPV $(56.60 \%)$. In patients who were $<30$ years old, the prevalence of MT HPV differed considerably from participants aged $30-44,45-59$ and $\geq 60$ years old. Thus, $64.02 \%$ of participants aged $<30$ years presented MT HPV, compared with $51.61 \%$ of participants aged $>29$ years old. Similar age-stratified proportions of ST and MT infections have been previously described in other countries $(38,40,41)$. This may be explained by differences in the immune system, and individuals may be capable of eradicating, at least in part, HPV infections over time.

In addition to the prevalence of specific HPV types, the present study analyzed the distribution of HPV types in participants with abnormal cervical cytology. Since HPV may cause the development of precancerous lesions (3), it was expected that the cytological findings from $\mathrm{HPV}^{+}$participants would be different to findings from $\mathrm{HPV}^{-}$participants. $\mathrm{HPV}^{+}$ participants exhibited higher grades of neoplasms (LSIL and HSIL) than HPV- participants. Furthermore, participants with $\geq 1$ HR-HPV type were more often diagnosed with HSIL than participants with LR-HPV. Similar observations concerning the association of poor cytological diagnosis with carcinogenic HPV types have been previously reported $(36,38)$. In all grades of cytological diagnosis, HPV16 was the most common HPV type (ASC-US, 29.46\%; LSIL, 29.58\%; HSIL, 43.22\%). In a similar study conducted previously in Italy (35), HPV16 was the most common HPV type observed in women with HSIL (27.30\%). However, in the present study, HPV51 and HPV56 had the highest incidence in participants with ASC-US lesions or LSIL (24.82 and $19.17 \%$, respectively). Future studies on which HPV type may become more important in the development of higher grade SILs as HPV vaccination coverage increases may be of great interest.

In the current study, 80 participants with cervical intraepithelial neoplasia were classified as grade CIN1 (22/80), CIN2 (27/80) or CIN3 (31/80). As previously reported, the incidences of particular HR-HPV infections were observed to increase in association with the severity of the cervical intraepithelial neoplasia in the present study $(5,35,42,43)$. In previous studies, each high-grade cervical neoplasia was attributed to a single HR-HPV infection, and HPV16 and 31 were the predominant HR-HPV types (44). These results are similar to the cytological findings of the present study, which demonstrated that the most frequent HPV types were HPV16 and 31 .

Although the present study revealed that the highest prevalence of HPV16 was in participants $<30$ years of age, it is important to emphasize that a subgroup of the youngest women, namely those aged $<23$ years old, exhibited a significantly lower incidence, compared with women aged 23-29 years old (28.57 vs. $45.71 \%$, respectively). The lower relative incidences of HPV16, 18 and 31 observed in the present study may be explained by a higher rate of vaccinated participants aged $<23$ years old. The low vaccination coverage in Germany $(45,46)$ may be attributed to the fact that women, who at the time of the present study were in their mid-twenties, were excluded from the recommendation for HPV vaccination, due to the restriction of the vaccination program to girls aged 12-17 years (47). In a recent study with a group of vaccinated women, the prevalence of HPV16 and 18 was significantly lower in women aged 20-21 years old, compared with non-vaccinated woman of the same age (48). The present study observed that the incidence of HPV31 was also lower in participants aged $<23$ years old, which may be due to the cross-protective effect of the two vaccines $(25,26)$. Since women that are aged $<23$ years old are more often protected against HPV16 and 18, as a result of vaccination programs that consist of girls and young women primarily, the proportion of other HPV types among infected women may gradually rise in future populations. Considering the increasing impact of vaccination against certain HPV types, it is important to acknowledge the shifting distribution of non-vaccine HPV types, predominantly HPV51, 53, 56 and 66, which were observed to have one of the highest incidences among the participants in the present study. A reduction in the incidence of HPV31, due to the cross-protective effects of the current vaccines, may also decrease the incidence of HPV16 and 18 with continuing or intensified vaccination programs. Although the present study expects that the proportion of HR-HPV types not covered by vaccination may increase in the near future, it remains unclear whether the absolute number of precancerous lesions and cervical cancer may also increase in the absence of HPV16 and 18. In a recently reported meta-analysis (27), HPV16 and 18 infections decreased between pre-vaccination and post-vaccination by $68 \%$ in girls aged $13-19$ years countries with a vaccination coverage of $\geq 50 \%$ (49), and the prevalence of HPV 31, 33 and 45 were also reduced. This probable effect of cross-protection was not reported for countries with a vaccination coverage of $<50 \%$ in girls aged $<20$-years-old, but a reduction in HPV16 and 18 was observed. Recently, it was demonstrated in a large cohort study that, due to the negative interaction of HPV16 with other HR-HPVs $(35,51$, 56 and 58), the removal of HPV16 may enable other HR-HPVs to become more prevalent (50). It was previously discussed that the relatively higher prevalence of HPV16 may result in under detection of low-copy HPV co-infections, particularly when the same amplification primers are used for HPV16 and other HPV types (50). Using an epidemiological approach, Tota et al (51) hypothesized that eradicating vaccine-targeted HPV16 and 18 enhances the chance of other HPV types not targeted by the vaccine to occupy the ecological niche created by the extinction of HPV16 and 18. Due to cross-protection against other HPV types with current vaccines and the upcoming implementation of novel multivalent vaccines against the majority of the HR-HPV types diminishes the risk of type replacement (52).

Consequently, adequate prevention strategies against HPV depend on adapted HPV testing, which should cover all non LR-HPV types. Notably, certain FDA-approved used HPV tests, such as Hybrid Capture 2 (Digene Corporation, Gaithersburg, MD, USA), do not cover all HR-HPV types, which were detected with the test platform used in the current study, including Papillocheck ${ }^{\circledR}$ (Greiner Bio One Ltd., Stonehouse, UK) and Linear Array ${ }^{\circledR}$ (Roche Diagnostics GmbH). The present results indicate that $9 \%$ of samples with HR-HPV infections may have been incorrectly reported as HPV by the Hybrid 
Capture 2 test. The information that eight HPV types formerly classified as low-risk or probably high-risk are indeed HR-HPV types increases the percentage of non-vaccine HR-HPV to higher values than previously expected (10). Accordingly, it may become increasingly important to monitor non-vaccine types using capable detection methods in future studies, and to investigate the possible increase of several HR-HPV types.

In participants from the present cohort study, high incidences of HR-HPV types (89.15\%) and a high incidence of HPV16 (32.98\%) were observed. In all grades of cytological diagnosis (ASC-US, LSIL and HSIL), HPV16 was the most common HPV type identified. The prevalence of specific HR-HPV infections increased with a more severe CIN grade. The number of findings of HR-HPV types, which may be suppressed directly (HPV16 and 18) or via cross-protection (HPV31) following vaccination, was considerably lower in participants aged $\leq 22$ years old (HPV16, 28.57\%; HPV18, 2.04\%; HPV31, 6.12\%), compared with those aged 23-29 years old (HPV16, 45.71\%; HPV18, 7.86\%; HPV31, 22.86\%). Consequently, in $\mathrm{HPV}^{+}$women of 16-22 years of age, who are more likely to be protected against certain HPV types by vaccination, compared with older woman, the present study observed an alteration in the incidences of HPV type. Consequently, future studies are required to investigate the risk of non-vaccine HR-HPV types for cervical cancer.

\section{Acknowledgements}

The authors of the present study would like to thank Mrs. Andrea Becher for using the data included in her dissertation thesis in the elaboration of the present manuscript. The authors acknowledge the support of all the staff members of the Part Shared Practice Molecular Pathology South Bavaria (Munich, Germany).

\section{References}

1. de Villiers EM, Fauquet C, Broker TR, Bernard HU and zur Hausen H: Classification of papillomaviruses. Virology 324: 17-27, 2004.

2. de Villiers EM: Cross-roads in the classification of papillomaviruses. Virology 445: 2-10, 2013.

3. Schiffman MH, Bauer HM, Hoover RN, Glass AG, Cadell DM, Rush BB, Scott DR, Sherman ME, Kurman RJ, Wacholder S, et al: Epidemiologic evidence showing that human papillomavirus infection causes most cervical intraepithelial neoplasia. J Natl Cancer Inst 85: 958-964, 1993.

4. Walboomers JM, Jacobs MV, Manos MM, Bosch FX, Kummer JA, Shah KV, Snijders PJ, Peto J, Meijer CJ and Muñoz N: Human papillomavirus is a necessary cause of invasive cervical cancer worldwide. J Pathol 189: 12-19, 1999.

5. Kjaer SK, van den Brule AJ, Bock JE, Poll PA, Engholm G, Sherman ME, Walboomers JM and Meijer CJ: Human papillomavirus - the most significant risk determinant of cervical intraepithelial neoplasia. Int J Cancer 65: 601-606, 1996.

6. Bosch FX, Lorincz A, Muñoz N, Meijer CJ and Shah KV: The causal relation between human papillomavirus and cervical cancer. J Clin Pathol 55: 244-265, 2002.

7. IARC: Latest World Cancer Statistics. Global cancer burden rises to 14.1 million new cases in 2012: Marked increase in breast cancers must be addressed. IARC/WHO Press Release Dec 223, 2013.

8. Robert Koch-Institute: Cancer in Germany 2009-2010: Incidences and Trends. 9th edition. Robert Koch Institute and the Association of Population-based Cancer Registries in Germany (eds). RKI-Hausdruckerei, Berlin, 2014

9. Burd EM: Human papillomavirus and cervical cancer. Clin Microbiol Rev 16: 1-17, 2003.
10. Halec G, Alemany L, Lloveras B, Schmitt M, Alejo M, Bosch FX, Tous S, Klaustermeier JE, Guimerà N, Grabe N, et al; Retrospective International Survey and HPV Time Trends Study Group; Retrospective International Survey and HPV Time Trends Study Group: Pathogenic role of the eight probably/possibly carcinogenic HPV types $26,53,66,67$, 68, 70, 73 and 82 in cervical cancer. J Pathol 234: 441-451, 2014.

11. Muñoz N, Bosch FX, de Sanjosé S, Herrero R, Castellsagué X, Shah KV, Snijders PJ and Meijer CJ; International Agency for Research on Cancer Multicenter Cervical Cancer Study Group: Epidemiologic classification of human papillomavirus types associated with cervical cancer. N Engl J Med 348: 518-527, 2003.

12. de Sanjose S, Quint WG, Alemany L, et al; Retrospective International Survey and HPV Time Trends Study Group: Human papillomavirus genotype attribution in invasive cervical cancer: A retrospective cross-sectional worldwide study. Lancet Oncol 11: 1048-1056, 2010

13. Schiffman M, Clifford G and Buonaguro FM: Classification of weakly carcinogenic human papillomavirus types: Addressing the limits of epidemiology at the borderline. Infect Agent Cancer 4: 8, 2009.

14. Clifford GM, Smith JS, Plummer M, Muñoz N and Franceschi S: Human papillomavirus types in invasive cervical cancer worldwide: A meta-analysis. Br J Cancer 88: 63-73, 2003.

15. Clifford GM, Gallus S, Herrero R, et al; IARC HPV Prevalence Surveys Study Group: Worldwide distribution of human papillomavirus types in cytologically normal women in the International Agency for Research on Cancer HPV prevalence surveys: A pooled analysis. Lancet 366: 991-998, 2005.

16. Smith JS, Lindsay L, Hoots B, Keys J, Franceschi S, Winer R and Clifford GM: Human papillomavirus type distribution in invasive cervical cancer and high-grade cervical lesions: A meta-analysis update. Int J Cancer 121: 621-632, 2007.

17. Lowy DR and Schiller JT: Prophylactic human papillomavirus vaccines. J Clin Invest 116: 1167-1173, 2006.

18. ECDC: Introduction of HPV vaccines in European Union countries - an update. ECDC, Stockholm, 2012.

19. Robert Koch-Institute: Recommendations of the Standing Committee on Vaccination (STIKO) at the Robert Koch Institute/Effective: August 2014. Epid Bull 34: 305-339, 2014

20. Paavonen J, Jenkins D, Bosch FX, et al; HPV PATRICIA study group: Efficacy of a prophylactic adjuvanted bivalent L1 virus-like-particle vaccine against infection with human papillomavirus types 16 and 18 in young women: An interim analysis of a phase III double-blind, randomised controlled trial. Lancet 369: 2161-2170, 2007.

21. Paavonen J, Naud P, Salmerón J, Wheeler CM, Chow SN, Apter D, Kitchener H, Castellsague X, Teixeira JC, Skinner SR, et al; HPV PATRICIA Study Group: Efficacy of human papillomavirus (HPV)-16/18 AS04-adjuvanted vaccine against cervical infection and precancer caused by oncogenic HPV types (PATRICIA): Final analysis of a double-blind, randomised study in young women. Lancet 374: 301-314, 2009.

22. FUTURE II Study Group: Quadrivalent vaccine against human papillomavirus to prevent high-grade cervical lesions. N Engl J Med 356: 1915-1927, 2007.

23. Lehtinen M, Paavonen J, Wheeler CM, et al: Overall efficacy of HPV-16/18 AS04-adjuvanted vaccine against grade 3 or greater cervical intraepithelial neoplasia: 4-year end-of-study analysis of the randomised, double-blind PATRICIA trial. Lancet Oncol 13: 89-99, 2012.

24. Tjalma WA: There are two prophylactic human papillomavirus vaccines against cancer, and they are different. J Clin Oncol 33: 964-965, 2015.

25. Malagón T, Drolet M, Boily MC, Franco EL, Jit M, Brisson J and Brisson M: Cross-protective efficacy of two human papillomavirus vaccines: A systematic review and meta-analysis. Lancet Infect Dis 12: 781-789, 2012.

26. Wheeler CM, Castellsagué X, Garland SM, et al; HPV PATRICIA Study Group: Cross-protective efficacy of HPV-16/18 AS04-adjuvanted vaccine against cervical infection and precancer caused by non-vaccine oncogenic HPV types: 4-year end-of-study analysis of the randomised, double-blind PATRICIA trial. Lancet Oncol 13: 100-110, 2012.

27. DroletM,BénardÉ,Boily MC,AliH,BaandrupL,BauerH,Beddows $\mathrm{S}$, Brisson J, Brotherton JM, Cummings T, Donovan B, et al: Population-level impact and herd effects following human papillomavirus vaccination programmes: a systematic review and meta-analysis. Lancet Infect Dis 15: 565-580, 2015. 
28. Plummer M, Vaccarella S and Franceschi S: Multiple human papillomavirus infections: the exception or the rule? J Infect Dis 203: 891-893, 2011.

29. Wagner D: Munich nomenclature II for gynaecologic cytodiagnosis. Acta Cytol 34: 900-901, 1990.

30. Solomon D, Davey D, Kurman R, Moriarty A, O'Connor D, Prey M, Raab S, Sherman M, Wilbur D, Wright T Jr, et al: The 2001 Bethesda System: Terminology for reporting results of cervical cytology. JAMA 287: 2114-2119, 2002.

31. Apgar BS, Zoschnick L and Wright TC Jr: The 2001 Bethesda System terminology. Am Fam Physician 68: 1992-1998, 2003.

32. WHO: WHO Guidelines: Guidelines for screening and treatment of precancerous lesions for cervical cancer prevention. World Health Organization, Geneva, 2013.

33. Cannavo I, Benchetrit M, Loubatier C, Michel G, Lemichez E and Giordanengo V: Characterization of a cluster of oncogenic mutations in E6 of a human papillomavirus 83 variant isolated from a high-grade squamous intraepithelial lesion. J Gen Virol 92: 2428-2436, 2011.

34. Robert Koch Institute: Vaccination against human papillomavirus among girls aged 12-17 years - recommendation and explanatory statement. Epidemiol Bull 12: 97-103, 2007 (In German).

35. Bruni L, Diaz M, Castellsagué X, Ferrer E, Bosch FX and de Sanjosé S: Cervical human papillomavirus prevalence in 5 continents: Meta-analysis of 1 million women with normal cytological findings. J Infect Dis 202: 1789-1799, 2010.

36. Meloni A, Pilia R, Campagna M, Usai A, Masia G, Caredda V and Coppola RC: Prevalence and molecular epidemiology of human papillomavirus infection in italian women with cervical cytological abnormalities. J Public Health Res 3: 157, 2014.

37. Klug SJ, Hukelmann M, Hollwitz B, Düzenli N, Schopp B, Petry KU and Iftner T: Prevalence of human papillomavirus types in women screened by cytology in Germany. J Med Virol 79: 616-625, 2007.

38. Iftner T, Eberle S, Iftner A, Holz B, Banik N, Quint W and Straube AN: Prevalence of low-risk and high-risk types of human papillomavirus and other risk factors for HPV infection in Germany within different age groups in women up to 30 years of age: An epidemiological observational study. J Med Virol 82: $1928-1939,2010$.

39. Argyri E, Papaspyridakos S, Tsimplaki E, Michala L, Myriokefalitaki E, Papassideri I, Daskalopoulou D, Tsiaoussi I, Magiakos G and Panotopoulou E: A cross sectional study of HPV type prevalence according to age and cytology. BMC Infect Dis 13: 53, 2013

40. Plummer M, Schiffman M, Castle PE, Maucort-Boulch D and Wheeler CM; ALTS Group: A 2-year prospective study of human papillomavirus persistence among women with a cytological diagnosis of atypical squamous cells of undetermined significance or low-grade squamous intraepithelial lesion. J Infect Dis 195: 1582-1589, 2007.

41. Heard I, Tondeur L, Arowas L, Falguières M, Demazoin MC and Favre M: Human papillomavirus types distribution in organised cervical cancer screening in France. PLoS One 8: e79372, 2013.
42. Resende LS, Rabelo-Santos SH, Sarian LO, Figueiredo Alves RR, Ribeiro AA,Zeferino LC and Derchain S: A portrait of single and multiple HPV type infections in Brazilian women of different age strata with squamous or glandular cervical lesions. BMC Infect Dis 14: 214, 2014

43. Cobo F, Concha A and Ortiz M: Human papillomavirus (HPV) type distribution in females with abnormal cervical cytology. A correlation with histological study. Open Virol J 3: 60-66, 2009.

44. García-Espinosa B,Moro-Rodríguez E and Alvarez-FernándezE: Genotype distribution of human papillomavirus (HPV) in histological sections of cervical intraepithelial neoplasia and invasive cervical carcinoma in Madrid, Spain. BMC Cancer 12: 533, 2012.

45. van der Marel J, Berkhof J, Ordi J, Torné A, Del Pino M, van Baars R, Schiffman M, Wentzensen N, Jenkins D and Quint WG: Attributing oncogenic human papillomavirus genotypes to high-grade cervical neoplasia: Which type causes the lesion? Am J Surg Pathol 39: 496-504, 2015.

46. Kuznetsov AV, Müller RA, Ruzicka T, Herzinger T and Kuznetsov L: Knowledge of sexually transmitted HPV infection, genitoanal warts, cancer and their prevention among young females after vaccine introduction in Germany. J Eur Acad Dermatol Venereol 27: 1527-1534, 2013.

47. Rieck T, Feig M, Deleré Y and Wichmann O: Utilization of administrative data to assess the association of an adolescent health check-up with human papillomavirus vaccine uptake in Germany. Vaccine 32: 5564-5569, 2014.

48. Deleré Y, Remschmidt C, Leuschner J, Schuster M, Fesenfeld M, Schneider A, Wichmann O and Kaufmann AM: Human Papillomavirus prevalence and probable first effects of vaccination in 20 to 25 year-old women in Germany: A population-based cross-sectional study via home-based self-sampling. BMC Infect Dis 14: 87, 2014.

49. Paul P and Fabio A: Literature review of HPV vaccine delivery strategies: Considerations for school- and non-school based immunization program. Vaccine 32: 320-326, 2014.

50. Yang Z, Cuzick J, Hunt WC and Wheeler CM: Concurrence of multiple human papillomavirus infections in a large US population-based cohort. Am J Epidemiol 180: 1066-1075, 2014.

51. Tota JE, Ramanakumar AV, Jiang M, Dillner J, Walter SD, Kaufman JS, Coutlée F, Villa LL and Franco EL: Epidemiologic approaches to evaluating the potential for human papillomavirus type replacement postvaccination. Am J Epidemiol 178: 625-634, 2013.

52. Safaeian M and Rodriguez AC: Invited commentary: Multiple human papillomavirus infections and type replacement-anticipating the future after human papillomavirus vaccination. Am J Epidemiol 180: 1076-1081, 2014. 